\title{
Thin low-loss dielectric coatings for free-space cloaking
}

\author{
Yaroslav Urzhumov, ${ }^{1, *}$ Nathan Landy, ${ }^{1}$ Tom Driscoll, ${ }^{1,2}$ Dimitri Basov, ${ }^{2}$ and David R. Smith ${ }^{1}$ \\ ${ }^{1}$ Center for Metamaterials and Integrated Plasmonics, Duke University, 130 Hudson Hall, P.O. Box 90271, \\ Durham, North Carolina 27708, USA \\ ${ }^{2}$ Physics Department, University of California San Diego, La Jolla, California 92093, USA \\ *Corresponding author: yaroslav.urzhumov@duke.edu
}

Received January 31, 2013; revised April 4, 2013; accepted April 10, 2013; posted April 11, 2013 (Doc. ID 184657); published May 3, 2013

\begin{abstract}
We report stereolithographic polymer-based fabrication and experimental operation of a microwave X-band cloaking device. The device is a relatively thin (about one wavelength thick) shell of an air-dielectric composite, in which the dielectric component has negligible loss and dispersion. In a finite band (9.7-10.1 GHz), the shell eliminates the shadow and strongly suppresses scattering from a conducting cylinder of six-wavelength diameter for TE-polarized free-space plane waves. The device does not require an immersion liquid or conducting ground planes for its operation. The dielectric constant of the polymer is low enough $(\epsilon=2.45)$ to suggest that this cloaking technique would be suitable for higher frequency radiation, including visible light. (c) 2013 Optical Society of America

OCIS codes: (160.5470) Polymers; (290.1350) Backscattering; (290.3200) Inverse scattering; (350.4010) Microwaves; (050.6624) Subwavelength structures; (290.2558) Forward scattering.

http://dx.doi.org/10.1364/OL.38.001606
\end{abstract}

The possibility to make large and nontransparent objects electromagnetically undetectable has been the subject of intense research following the introduction of the transformation optics (TO) concept $[1,2]$ and its microwave implementation [3]. In theoretical TO scenarios, an inhomogeneous shell around an object eliminates backward reflection and forward shadow, thus by virtue of the optical theorem also suppressing the scattering in all other directions.

Currently, however, no technique is available for fabrication of invisibility devices capable of hiding objects with cross sections exceeding 5 free-space wavelengths $(\lambda)[4]$. Inherent in TO-based proposals are exotic material properties, such as large or near-zero magnetic permeability and/or dielectric permittivity, both with negligible imaginary parts. To date, virtually all TO medium proposals involved metallic components behaving as conducting [3-5] or plasmonic [6,7] media. Propagation loss is a major limitation for cloaks already at microwave frequencies [3,4], and it becomes overwhelming at visible wavelengths.

The attenuation issue prompted a search for invisibility scenarios that could be based entirely on low-loss dielectric materials with a dielectric constant $\epsilon<1$. Naturally birefringent dielectric crystals such as calcite $[\underline{8}, \underline{9}]$ were used in visible-wavelength cloaking experiments. Having refractive indexes $n_{o, e}>1$, such media are only capable of cloaking if submerged in a high-index immersion liquid whose refractive index $n_{\text {im }}$ satisfies $1<n_{o / e}<n_{\text {im }}$. Immersion helps avoid the most fundamental limitation of TO-based cloaking - the need for superluminal phase velocities $[\underline{10}, \underline{11}]$ - while rendering these cloaks useless for free-space applications such as sensing countermeasures.

In this Letter, we present a general design and fabrication methodology that yields 2D microwave cloaks composed of only one, virtually lossless, dispersionless, dielectric medium with $\epsilon=2.45$. The cloaks are multidirectional, i.e., invisible with respect to several propagation directions, are capable of hiding objects many wavelengths in diameter, and are thinner relative to the object size than in any previous experimental demonstrations [ $\underline{3}-\underline{5}]$. Here, we apply the technique to TE-polarization waves, however, it also yields cloaks with similar performance metrics for TM polarization. Our choice of TE polarization is dictated by the testing apparatus, but it is not a limitation of the design approach [12].

We take a one-step shape-and-topology optimization approach $[12,13]$ where the distribution of a single material is optimized in the entire structure without constraining the number, location, or shape of voids. The resulting structure can be easily fabricated using rapid prototyping techniques, also known as stereolithographic (STL) fabrication or 3D printing. To ensure easy fabrication and structural integrity of the cloak, we only consider solutions with a sufficiently low concentration of voids, in which the dielectric forms a single, contiguous piece.

For fabrication, we use Fused Deposition Modeling, one of the polymer-based STL techniques, with ABS plus (acrylonitrile butadiene styrene) as the model material [Fig. 1(a)]. To ensure accurate design of the cloak, we performed in-house characterization of ABS supplied by Stratasys [Fig. 1(b)]. We accomplished this by loading a rectangular waveguide (WR-90 standard) with a $120 \mathrm{~mm}$ length of plastic and measuring the phase delay in transmission in the X-band (8-12 GHz). The best-fit
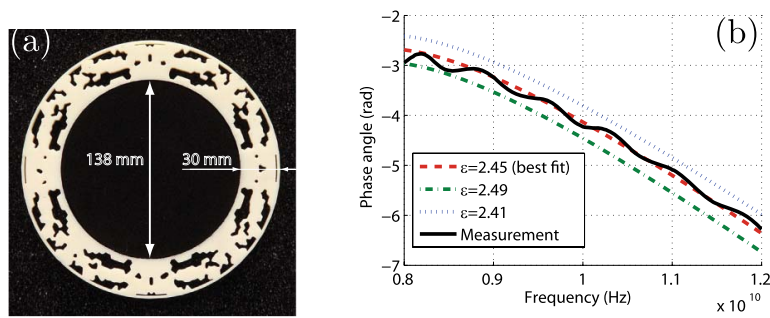

Fig. 1. (a) Photograph of the fabricated dielectric cloak; outer diameter $198 \mathrm{~mm}$, inner diameter $138 \mathrm{~mm}$. Aluminum foil annulus and an absorbing disk matching the inner diameter of the cloak are inserted for measurements. (b) ABS characterization: phase advance versus frequency in the X-band for a planar material slab of $120 \mathrm{~mm}$ thickness. 
dielectric constant, $\epsilon=2.45$ (with zero loss tangent) provides an excellent fit in the entire X-band, indicating negligible dispersion and attenuation in the ABS. While our procedure is not sufficiently sensitive to put a lower bound on the dielectric loss, subsequent measurements of the ABS-based cloak confirm that the attenuation in ABS is negligible at $10 \mathrm{GHz}$ on the 8-in. spatial scale of our device.

Once the accurate dielectric constant was determined, we have designed a low-scattering structure (approximate cloak) using a numerical optimization algorithm fitted to the capabilities of the modeling package COMSOL Multiphysics [14]. For the forward solver, we use the frequency-domain scattered-field solver in the RF Module of COMSOL, with a monochromatic plane wave as the background field (the excitation). Scattered field is computed in a simulation domain consisting of the cloak $(\Omega)$ and surrounding free space, which is terminated with an absorbing boundary at radius $R=3 R_{\text {cloak }}$, as seen in Fig. 2(a). The shape of the model material is described using a level-set function, whose values are discretized on a finite-element mesh and treated as optimization variables (unknowns). In contrast with a related method [15], which was the first method to yield dispersionlessdielectric cloaks, we do not assume a continuous distribution of permittivity: our dielectric composite is binary and thus, can be immediately translated into a machinable design. This method is described in some detail in Ref. [12]; here, we summarize only the key features.

The shape of the model material domain $\Omega_{m}$ is discretized as follows. An auxiliary scalar field variable $p(x, y)$ is introduced in the entire cloak domain $\Omega$; its values are restricted to the closed interval $p \in[-1,1]$. The field $p$ enters the EM equations through the locally isotropic dielectric constant $\epsilon=\epsilon_{a}+\left(\epsilon_{m}-\epsilon_{a}\right) \theta_{\delta}(p)$, where $\theta_{\delta}(p)$ is a smoothed Heaviside function with smoothing interval $2 \delta$, i.e., $\theta_{\delta}(p)=0$ for $p<-\delta$ and $\theta_{\delta}(p)=1$ for $p>\delta$. The embedded optimization algorithm in COMSOL makes use of the adjoint method to compute the gradient of the cost function with respect to all optimization variables. We define the cost function as the integral of the norm of scattered field over the exterior boundary of the simulation domain, the quantity we term near-field scattering cross section (SCS) [Fig. 2(b)].

To further reduce computational cost, we introduce $N$ symmetry planes separated by angles $\pi / N$ and intersecting at the origin. The resulting structure has $C_{N v}$ symmetry and is, therefore, a multidirectional cloak with reduced scattering for waves incident from $N$ distinct directions. Our algorithm converged to a strongly cloaked

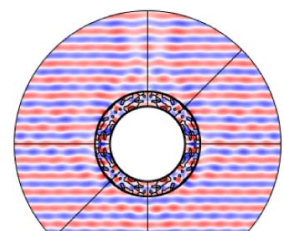

(a)

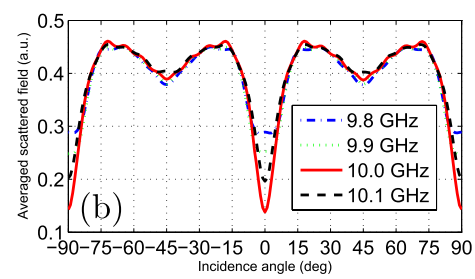

incidence angle (deg)
Fig. 2. (a) Single-dielectric multidirectional cloak simulated at $10 \mathrm{GHz}$ frequency, with a plane wave incident along the symmetry plane. (b) Near-field scattering cross section (SCS) versus incidence angle. solution for $N=1,2,4$ and 8; similar limitations on $N$ were observed in Ref. [15]. The optimization process for $N=4$ carried out at a single frequency $(10 \mathrm{GHz})$ yielded the structure shown in Figs. 2(a) and 2(b). The contours of the dielectric subdomain were vectorized and extruded out of plane to create a 3D CAD description (STL format) of the subsequently fabricated structure [Fig. 1(a)]. Holes smaller than $0.8 \mathrm{~mm}$ (roughly the minimum wall size attainable with our FDM machine) were neglected and eliminated.

We characterized the fabricated cloak in our 2D mapping chamber. This chamber consists of a parallel-plate waveguide region terminated on all sides by microwave absorber, and emulates free-space propagation for TE waves [16]. The incident beam was formed by collimating a point-source excitation with a hyperbolic lens [17] milled from a sheet of polycarbonate. The presence of a moving part of the field-scanning apparatus required us to implement an air gap between the sample and the top plate of the waveguide. In the absence of such a gap, the behavior of guided TEM waves mimics the scattering of a TE-polarized plane wave off an infinitely tall sample. In the design process, we accounted for the effect of the air gap approximately by using the effective dielectric constant [18] $\epsilon_{\text {eff }}=((1-f)$ $\left.\epsilon_{\mathrm{ABS}}^{-1}+f\right)^{-1}$, where $f$ is the ratio of gap size to the height of waveguide.

Thus measured E-field amplitude [Figs. 3(e)-3(h)] and E-field intensity maps [Figs. 4(a) and 4(b)] show that the system composed of the dielectric composite shell and a conducting cylinder has a negligible shadow. This is particularly clear in comparison both with a bare cylinder [Fig. 4(c)] and a PEC cylinder coated with a solid ABS layer of the same outer diameter as the cloak [Fig. 4(d)]. Moreover, the geometrical shadow zone is populated by a plane wave whose phase fronts are aligned with the phase fronts outside the shadow.

To quantify the degree by which the dielectric shell reduces scattering and determine the bandwidth of this phenomenon, we have calculated the magnitude of scattered electric field spatially averaged over a contour in the Fresnel zone of the structure [Fig. 5(a)]. Both experimental data and simulations suggest that the structure operates as a cloak in the $9.7-10.1 \mathrm{GHz}$ band. Visibility of the structure is reduced at optimal frequency by as much as 4 times relative to an uncloaked cylinder, and 5 times relative to a PEC cylinder coated with a solid ABS shell of the same thickness as the cloak. A
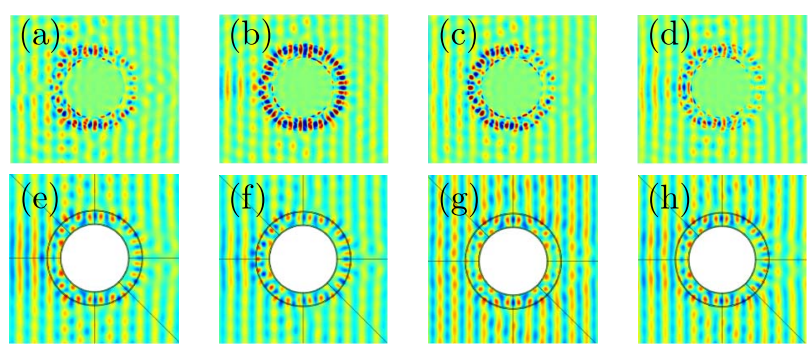

Fig. 3. Electric field maps at several frequencies (GHz): (a) 9.7 , (b) 9.9 , (c) 10.0, and (d) 10.1. Second row: simulation results at the corresponding frequencies. 

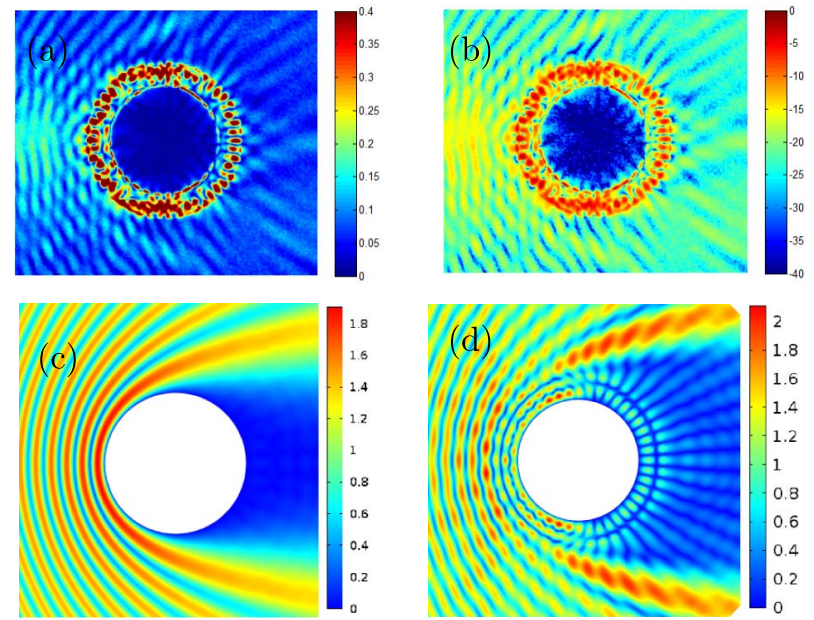

Fig. 4. Field intensity distributions around the cloaked cylinder measured at $9.9 \mathrm{GHz}$ : (a) linear scale and (b) logarithmic intensity scale. Second row: field intensity (linear scale) for an uncloaked PEC cylinder of the same diameter, and a PEC cylinder cloaked with a homogeneous dielectric coating of the same thickness as the cloak (simulations).
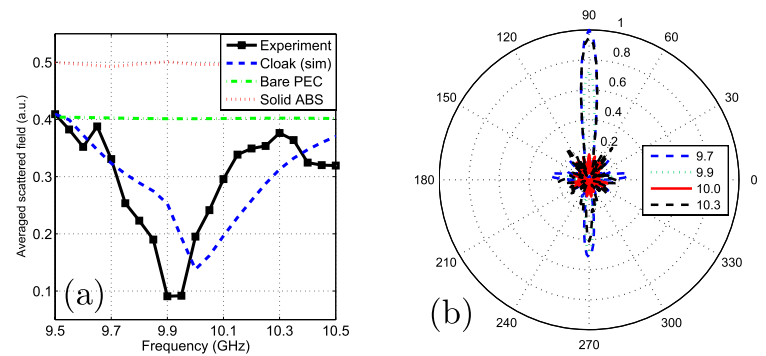

Fig. 5. (a) Spatially-averaged scattered field in the Fresnel zone (experiment and simulation) versus frequency. For comparison, dotted and dash-dotted curves show the same figure of visibility for a bare PEC cylinder and a PEC cylinder coated with solid ABS of same thickness as the cloak. (b) Far field of the cloaked system at different frequencies in the 9.7$10.3 \mathrm{GHz}$ range versus polar angle $\left(\phi=90^{\circ}\right.$ corresponds to the $y$ axis and the incidence direction).

calculation of the far field via the Kirchhoff integral [Fig. 5(b)] confirms that the cloak has strongly reduced visibility in the Fraunhofer diffraction zone as well.
The close agreement between numerical simulations and field measurements proves that lossless all-dielectric binary composites can reconstruct a plane wave-with an unperturbed magnitude and phase-in the geometrical shadow region of a large, nontransparent object spanning many wavelengths in diameter. Given the relatively low refractive index of the dielectric component $(n=1.56)$, there are no physical limitations to translating this technique to higher, including optical, frequencies.

This work was supported by the U.S. Army Research Office through a Multidisciplinary University Research Initiative (Grant No. W911NF-09-1-0539).

\section{References}

1. J. Pendry, D. Schurig, and D. R. Smith, Science 312, 1780 (2006).

2. Y. A. Urzhumov, N. B. Kundtz, D. R. Smith, and J. B. Pendry, J. Opt. 13, 024002 (2011).

3. D. Schurig, J. J. Mock, B. J. Justice, S. A. Cummer, J. B. Pendry, A. F. Starr, and D. R. Smith, Science 314, 977 (2006).

4. N. Landy and D. R. Smith, Nat. Mater. 12, 25 (2012).

5. S. Tretyakov, P. Alitalo, O. Luukkonen, and C. Simovski, Phys. Rev. Lett. 103, 103905 (2009).

6. W. Cai, U. K. Chettiar, A. V. Kildishev, and V. M. Shalaev, Nat. Photonics 1, 224 (2007).

7. A. Alu and N. Engheta, Phys. Rev. Lett. 100, 113901 (2008).

8. X. Chen, Y. Luo, J. Zhang, K. Jiang, J. B. Pendry, and S. Zhang, Nat. Commun. 2, 176 (2011).

9. B. Zhang, Y. Luo, X. Liu, and G. Barbastathis, Phys. Rev. Lett. 106, 033901 (2011).

10. Y. A. Urzhumov and D. R. Smith, Phys. Rev. Lett. 105, 163901 (2010).

11. Y. A. Urzhumov and D. R. Smith, Opt. Lett. 37, 4471 (2012).

12. J. Andkær, N. A. Mortensen, and O. Sigmund, Appl. Phys. Lett. 100, 101106 (2012).

13. M. P. Bendsøe and O. Sigmund, Topology Optimization: Theory, Methods and Applications (Springer, 2003).

14. COMSOL AB, COMSOL Multiphysics User's Guide, Version 4.2a (Burlington, 2011).

15. J. Andkær and O. Sigmund, Appl. Phys. Lett. 98, 021112 (2011).

16. B. Justice, J. J. Mock, L. Guo, A. Degiron, D. Schurig, and D. R. Smith, Opt. Express 14, 8694 (2006).

17. T. A. Milligan, Modern Antenna Design, 2nd ed. (Wiley, 2005).

18. D. E. Aspnes, Am. J. Phys. 50, 704 (1982). 